\title{
USO DEL LÁSER DE DIODO COMO COADYUVANTE EN EL TRATAMIENTO DE PERIODONTITIS CRÓNICA.
}

\section{USE OF THE DIODE LASER AS A COADYUVANT IN THE CHRONIC PERIODONTITIS TREATMENT.}

\author{
Casadoumecq, Ana Clara ${ }^{1}$ \\ ${ }^{1}$ Cátedra de Fisiología y Coordinadora Unidad Láser, Facultad de Odontología, Universidad de Buenos Aires. \\ *anaclaracasa64@yahoo.com.ar
}

\begin{abstract}
Resumen
Se presenta en este manuscrito una revisión actualizada del estado del arte acerca de las características y el uso terapéutico de láser de diodo. La información presentada sintetiza las evidencias experimentales que indican la aplicación de este tipo de láser, como co-adyuvante en el tratamiento periodontal, permite obtener mejores índices periodontales en la reevaluación del paciente. Por lo tanto, el empleo de esta tecnología podría reducir la necesidad de tratamiento quirúrgico convencional adicional
\end{abstract}

Palabras clave: Láser de diodo, terapia periodontal no quirúrgica, peridontitis crónica.

\begin{abstract}
An updated review of the state of the art describing the characteristics and the therapeutic use of the diode laser in the dental field is presented in this manuscript. The information provided summarizes the experimental and clinical evidences that indicate that the application of this type of laser, cosidered as coadjuvant in the periodontal treatment, allows to obtain better periodontal indexes in the re-evaluation of the patient. Therefore, the use of this tecnology may reduce the need for additional conventional surgical treatment.
\end{abstract}

Key words: Diode laser, surgical periodontal therapy, chronic periodontal disease.

\section{INTRODUCCIÓN}

En 1917, Albert Einstein daba a conocer al mundo una de sus publicaciones más importantes sobre el proceso de emisión de radiación estimulada "The Quantum Theory of Radiation", dando inicio a la ley del efecto fotoeléctrico. La palabra Láser (del acrónimo inglés LASER, Light Amplification by Stimulated Emission of Radiation) significa amplificación de luz por emisión de radiación estimulada. A través de un razonamiento teórico se creó la base física más importante del láser: el proceso de emisión estimulada, ${ }^{1}$ Según Bayat y col. ${ }^{2}$ y Barolet y col. ${ }^{3}$ la estimulación con láser se usa ampliamente para aplicaciones médicas debido a su naturaleza de radiación electromagnética, dado que, al no pertenecer a la familia de rayos $\mathrm{X}$, no posee efecto ionizante sobre los tejidos. En tal sentido, el láser ha sido utilizado en muchas especialidades médicas, entre ellas en la odontología. Una de las aplicaciones de este tipo de tecnología es en la terapia de la enfermedad periodontal. Sin embargo, a pesar de la aparente utilidad del láser, ¿por qué después de casi tres décadas, el uso del láser en la terapia periodontal sigue siendo controvertido? ¿Es porque el láser desafía las modalidades tradicionales de tratamiento de la periodontitis o por la falta de pruebas sólidas para tomar una decisión informada? En la práctica privada de la profesión se están usando varios tipos de láser para el tratamiento de la enfermedad periodontal y la mayoría de profesionales que lo emplean ha expresado su satisfacción con los resultados obtenidos. Sin embargo, revisiones sistemáticas recientes han sugerido que hay poca evidencia que respalde los supuestos beneficios del láser en el tratamiento de la enfermedad periodontal en comparación con la terapia periodontal tradicional. ${ }^{4,5}$ Por lo tanto surge la necesidad de saber si el uso de láser en periodoncia se basa en la evidencia publicada-revisada por pares y obtenida bajo condiciones controladas- o en la evidencia no confirmada transmitida de boca en boca. Ante esta situación, el presente artículo de revisión pretende responder a la pregunta: ¿Existe evidencia actual en relación con la efectividad del uso del láser como co-adyuvante en el tratamiento de la periodontitis? 


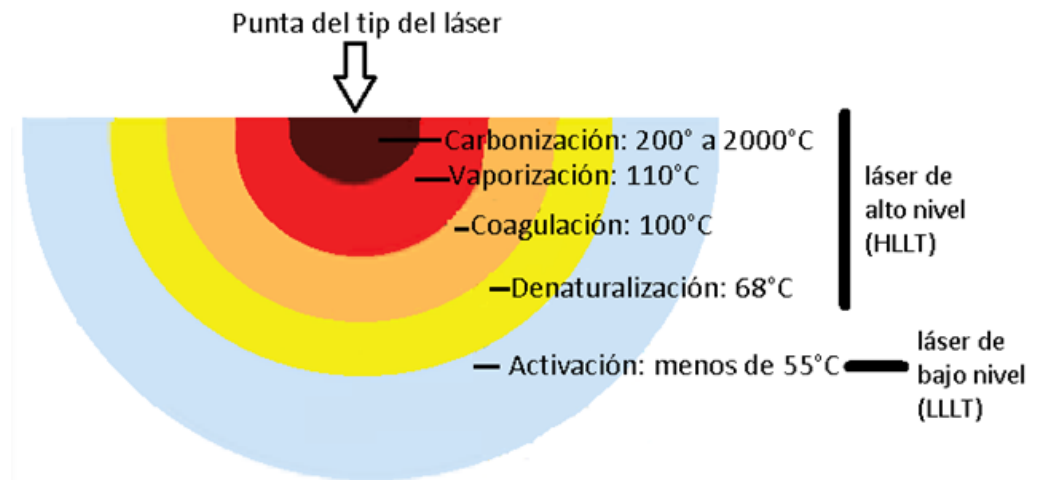

Fig. 1. Terapia con láser de alto nivel (HLLT) y terapia con láser de bajo nivel (LLLT). El tratamiento con láser de alto nivel puede causar diversos grados de efectos térmicos en los tejidos, incluida la coagulación y la ablación de los tejidos blandos y la eliminación de los tejidos duros. Simultáneamente, un nivel bajo de energía penetra o se dispersa en los tejidos circundantes durante el tratamiento con láser de alto nivel. El tratamiento con láser de bajo nivel estimula los tejidos / células sin producir cambios térmicos irreversibles en los tejidos, lo que resulta en la activación o estimulación (fotobiomodulación) de la cicatrización de heridas en los tejidos circundantes. Cuando se utiliza un láser de alto nivel con un nivel de energía bajo, el efecto térmico también puede inducir la cicatrización de la herida, como en la fotobiomodulación de efecto del láser de nivel puramente bajo (PBM) (Adaptado de Aoki y col., 2015).

\section{ESTADO DEL ARTE}

Las características clave de la luz láser son su monocromaticidad (que está compuesta de un solo color o longitud de onda), su coherencia (todas las ondas de luz están espacial y temporalmente en fase) y su colimación (todos los rayos de luz son paralelos y no divergen significativamente, incluso en largas distancias). Estas propiedades, asociadas al tipo de tejido diana, son esenciales para la elección de la fuente de láser a emplear en un determinado tratamiento. ${ }^{6}$

El láser interactúa de diferente forma con los tejidos, determinando los siguientes procesos: ablación o vaporización, hemostasia, inhibición, destrucción microbiana, y bioestimulación (fotobiomodulación o Low level laser therapy - LLLT). Mediante la aplicación del láser se inducen diversos efectos terapéuticos beneficiosos y respuestas biológicas que dependen de una gran variedad de parámetros, como la longitud de onda, la irradiancia, el tiempo, la coherencia de la luz y la polarización, el área tratada y el cromóforo en interacción. La evaluación crítica de la interacción entre estos factores representa un gran desafío, tanto para los investigadores como para los clínicos. ${ }^{6}$

Según la primera ley de fotobiología, la absorción de luz requiere la presencia de un cromóforo, es decir, un compuesto o molécula que absorbe la luz a una determinada longitud de onda. Por su parte, dependiendo de su longitud de onda, existen dos tipos de láser: (i) de penetración profunda — como el láser de diodo $(475 a 980 \mathrm{~nm})$ y el láser de NdYAG o granate de itrio y aluminio dopado con impurezas de neodimio (1064nm) - que cuando se emplean en tejidos blandos, su luz penetra y se dispersa profundamente; (ii) de penetración superficial - como el láser de dióxido de carbono $(10600 \mathrm{~nm})$, Er: YAG $(2940 \mathrm{~nm})$ y Er, Cr: YSGG (2780nm) - que cuando se aplican en tejidos duros como el hueso o la hidroxiapatita, su luz es absorbida en la capa superficial y no penetra ni se dispersa profundamente. ${ }^{7}$

La fluencia que emite un láser es la densidad de energía y está en relación con la potencia (energía) que emite el láser y se mide en vatios, el tiempo t (en milisegundos) y la superficie irradiada (área del círculo irradiada), S, por el haz de luz láser. Esta fluencia se mide en joules = vatios $/ \mathrm{cm} 2$. Es el dato principal para poder comparar trabajos porque indica cuanta energía se aplicó aun utilizando fibras de distintos diámetros $(\mu \mathrm{m})$.

Chaves y col. ${ }^{8}$ indican que la dosis de láser que se administra a los pacientes durante un tratamiento se basa en la densidad de energía, medida en unidades de Joules (J) por centímetro cuadrado $\left(\mathrm{J} / \mathrm{cm}^{2}\right)$ o simplemente energía expresada como Joules. Pocos dispositivos utilizados en la terapia con láser permiten el ajuste de la dosis en $\left(\mathrm{J} / \mathrm{cm}^{2}\right)$ o J. Por otra parte, la densidad de energía que se ha demostrado como efectiva en la inducción de efectos biológicos positivos (bioestimulación) varía de 0,09 a $90 \mathrm{~J} / \mathrm{cm} 2$; sin embargo, la más utilizada y efectiva varía entre 1 y $5\left(\mathrm{~J} / \mathrm{cm}^{2}\right)$.

Según la forma de aplicación de la dosis de energía, los láseres pueden clasificarse como terapéuticos o quirúrgicos. Por ejemplo, dependiendo de la dosis con la que se aplique el láser de diodo, este provocará regeneración del tejido o su vaporización. Aoki y col. ${ }^{7}$ destacan que el láser ha estado disponible para su uso en odontología desde 1989, pero que no ha sido aceptado universalmente. De hecho, como mencionamos anteriormente, su eficacia para ciertos procedimientos dentales, como la terapia periodontal no quirúrgica, todavía es controversial.

Dentro de las bolsas periodontales, el biofilm bacteriano y sus endotoxinas colonizan las superficies radiculares, penetran en el cemento radicular y en la pared blanda de la bolsa. 
Es esencial eliminar el biofilm por completo para restablecer el equilibrio inmunológico y permitir una nueva fijación del periodonto. Sin embargo, debido a la complejidad anatómica, la desinfección completa y el desbridamiento de las bolsas periodontales no siempre pueden lograrse con la terapia mecánica convencional. La mayoría de los tipos de láser quirúrgico son capaces de eliminar las bacterias por efectos fototérmicos (efecto bactericida). Además, el tratamiento con láser dentro de la bolsa aumenta el sangrado de la superficie del hueso desbridado en las bolsas y defectos óseos, lo que resulta ventajoso para la regeneración de los tejidos. ${ }^{4}$

El modelo explicativo actual de las enfermedades gingivo periodontales inducidas por placa incluye un componente microbiano patógeno, una respuesta inflamatoria del huésped y diversos factores de riesgo que contribuyen a la susceptibilidad y progresión de la enfermedad. Teniendo en cuenta el componente microbiano, parece lógico que la irradiación con láser, por su efecto bactericida, tenga un potencial significativo como alternativa o complemento de la terapia tradicional no quirúrgica. ${ }^{9}$

Al momento de seleccionar un protocolo de aplicación de láser se debe tener claro cómo se utilizó, por cuánto tiempo, si se utilizó o no fibras para llegar a la profundidad de la bolsa, o si se repitió el procedimiento. De hecho, para poder comparar los resultados debemos conocer los parámetros que se utilizaron. Se realizó una revisión de literatura en las bases digitales PUBMED, Scielo y Scopus, para presentar una compilación de investigaciones que reportan las indicaciones de los distintos tipos de láser, sus características y el procedimiento indicado (Figura2).

\section{DISCUSIÓN}

Pamuk y col. ${ }^{10}$, Gündoğar y col. ${ }^{11}$ y Roncati y col. ${ }^{12}$ utilizaron la terapia láser de baja potencia siempre acompañada por el tratamiento convencional de Raspaje y Alisado (RyA). Las longitudes de onda utilizadas variaron entre 685 y $980 \mathrm{~nm}$, lo cual dificulta la comparación de protocolos porque cada longitud de onda se utiliza con diferentes parámetros, Aun cuando emplearon la misma longitud de onda, $(980 \mathrm{~nm})^{13}$ Fenol y col, ${ }^{13}$ Dukić $^{14}$ y Deepa Raj ${ }^{15}$ utilizaron diferente diámetro de fibra, tiempo y potencia.

Para evitar esta imposibilidad de comparación es que se debiera considerar la densidad de potencia y la fluencia utilizada, que son parámetros que se pueden calcular trabajando con diferentes diámetros de fibra o tiempo. Balasubramaniam $\mathrm{y} \mathrm{col}^{16}$ es el único que indica ambos parámetros.

El tiempo de aplicación coincide entre 20 y 30 segundos, pero no es lo mismo irradiado en una profundidad de sondaje (PS) de más de $7 \mathrm{~mm}$ que una PS de $4 \mathrm{~mm}$. Thakare Kaustubh y col. ${ }^{17}$ reportan que calculan segun la PS pero no indican como.

En su estudio a dos meses, Fenol y col. ${ }^{13}$ hallaron que el sitio de prueba donde se usó el láser como coadyuvante mostró una reducción significativa en la profundidad del sondaje, nivel de inserción clínica, índice gingival (IG) y patógenos periodontales, lo que muestra que la cantidad de recolonización de carga microbiana es menor cuando se usa el láser como coadyuvante de la terapia convencional. A los 60 días, ${ }^{18}$ detectaron mejoras estadísticamente significativas en IG, PS, nivel de inserción clínica y IGS para el grupo RyA + diodo en comparación con el grupo RyA, pero no hubo diferencias significativas en la PI entre los grupos. Además, el porcentaje de sitios positivos para $\mathrm{P}$. gingivalis en el grupo RyA + diodo disminuyó del $80 \%(12 / 15)$ al $20 \%$ (3/15) después de la irradiación con láser $(\mathrm{p}<0.05)$. No observaron cambios significativos en el grupo de RyA.

Al comparar los resultados inmunohistoquímicos, Saglam y col. ${ }^{19}$ observaron que el grupo de pacientes tratados con láser mostró un resultado significativamente mejor en comparación con el grupo control en relación con los parámetros clínicos de boca completa. MMP-1, MMP-8 y TIMP-1 mostraron diferencias significativas entre los grupos después del tratamiento en comparación con la línea de base ( $\mathrm{p}<0.05)$. La cantidad total de $I L-1 \beta, I L-6, M M P-1$, $M M P-8$ y $T I M P-1$ disminuyó $(p<0,05)$ y la $I L-8$ aumentó después del tratamiento en los grupos de láser y control $(p<0,05)$.

En cambio, Gündoğar y col ${ }^{11}$ no hallaron las diferencias en los niveles de citoquinas GCF entre los grupos. Sin embargo, en el primer mes los niveles de PS fueron significativamente más bajos $(p<0,05)$ en el grupo de $R y A+L L L T$ que en el grupo de RYA. En el tercero y sexto meses, el nivel de inserción clínica, PS y IG fueron significativamente más bajos $(p<0,05)$ en el grupo de $R Y A+L L L T$ que en el grupo de RyA.

Para Dukić ${ }^{14}$ los resultados fueron similares para ambos grupos en términos de IP, SS, PS profundas y nivel de inserción clínica. El grupo de pacientes tratados con láser mostró solo una ganancia significativa de PS en bolsas moderadas durante el período de referencia a períodos de 18 semanas $(p<0,05)$ y de 6 a 18 semanas $(p<0,05)$, mientras que no se encontraron diferencias entre el grupo tratado con láser y el grupo control en relación con el resto parámetros $(p>0,05)$.

Roncati y col. ${ }^{12}$ encontraron mejores resultados en piezas dentarias multiradiculares. En el grupo láser, las PS de las piezas multiradiculares disminuyeron de $6.2 \mathrm{~mm}$ a 3.3 mm después de 1 año, y se mantuvieron estables durante 10 años. El aumento medio del nivel de inserción clínica fue de $2.2 \mathrm{~mm}$ en este grupo. En el grupo control, las PS disminuyeron de $7 \mathrm{~mm}$ a $5.8 \mathrm{~mm}$ después de 1 año y fueron de $5.1 \mathrm{~mm}$ después de 10 años. El incremento medio en nivel de inserción clínica fue de $1.9 \mathrm{~mm}$. En términos generales, el grupo de pacientes tratados con láser presentó parámetros clínicos significativamente mejores que los del grupo control, incluida la reducción de la pérdida de dientes. 


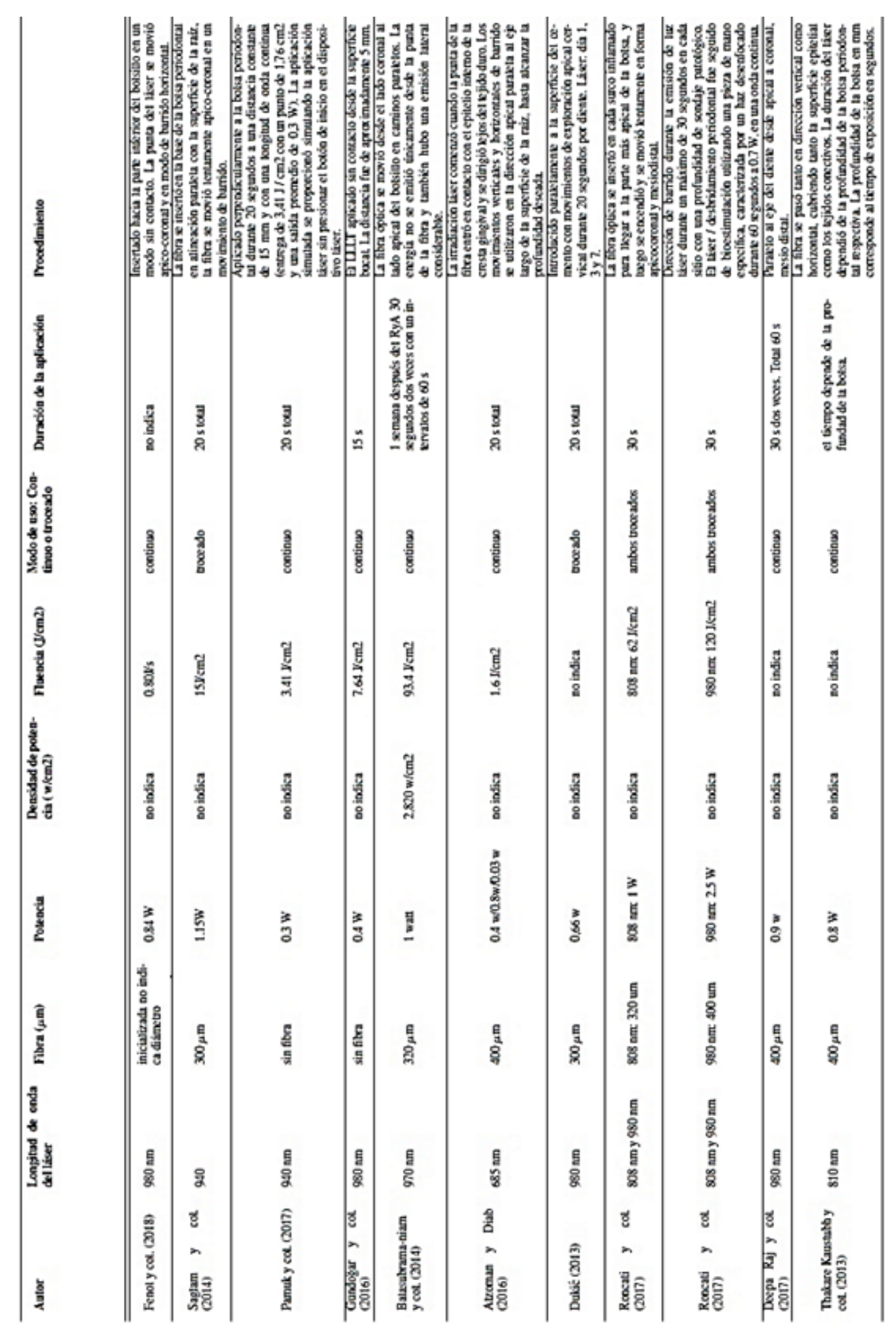

Fig. 2. Características de láser empleado y procedimiento utilizado 


\section{CONCLUSIONES}

Si bien para poder avanzar la ciencia necesita estar abierta a nuevas modalidades de tratamiento, las mismas deben estar basadas en protocolos que hayan sido probados en los diferentes niveles de evidencia. Para poder comparar trabajos o reproducirlos es necesario estandarizar parámetros que se puedan adaptar a las mismas familias de láseres, en este caso de diodo. Aunque los resultados siguen siendo controversiales, en PS moderadas se observa una tendencia a producirse un efecto beneficioso. Para zanjar la controversia es necesario mejor sustento básico en modelos animales, antes de extrapolar los resultados a la aplicación clínica incorporando variables como la acción del cigarrillo o enfermedades de base, dado que la mayoría de los pacientes con los que tratamos en la práctica diaria poseen alguna característica sistémica, endógena o exógena, que puede interferir con el resultado de la acción terapéutica.

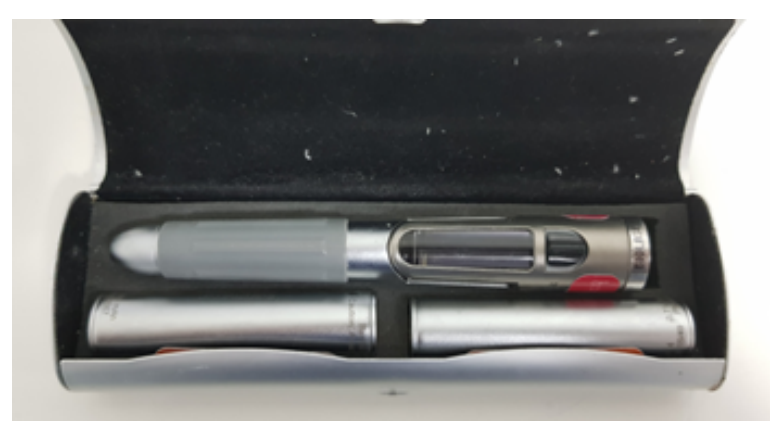

Fig. 3. Equipo láser 940nm modelo Ilase marca Biolas.

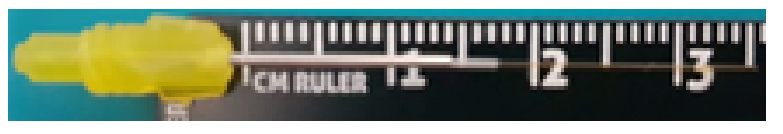

Fig. 6. Tip endodontico de diámetro de $200 \mu \mathrm{m}$.

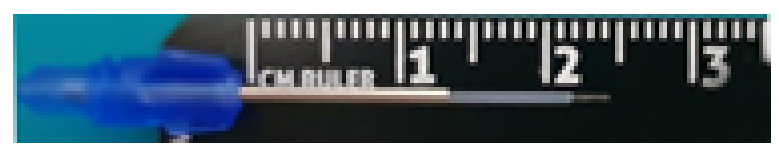

Fig. 7. Tip periodontal de diámetro de $300 \mu \mathrm{m}$.

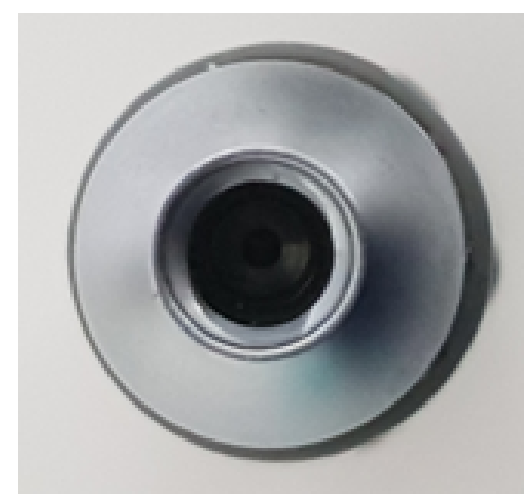

Fig. 4. Área Spot. Lugar de salida del láser de diámetro de $0,1 \mathrm{~cm}^{2}$.

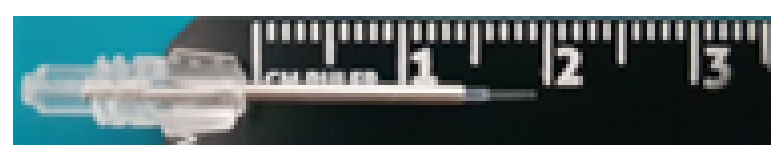

Fig. 5. Tip quirúrgico de diámetro de $400 \mu \mathrm{m}$. 
CONFLICTO DE INTERESES: La autora no 13 Fenol A, Boban NC, Jayachandran P, Shereef M, Balakrish-

manifiesta ningún conflicto de interés.

\section{Referencias Bibliográficas}

1 Einstein A. Zur quantentheorie der strahlung. Phys Z. 1917;18:121-128.

2 Bayat M, Virdi A, Jalalifirouzkouhi R, Rezaei F. Comparison of effects of LLLT and LIPUS on fracture healing in animal models and patients: A systematic review. Progress in biophysics and molecular biology. 2018;132:3-22.

3 Barolet D, Christiaens F, Hamblin MR. Infrared and skin: Friend or foe. Journal of Photochemistry and Photobiology B: Biology. 2016;155:78-85.

4 Cobb CM. Lasers in periodontics: a review of the literature. Journal of periodontology. 2006;77(4):545-564.

5 Qadri T, Javed F, Johannsen G, Gustafsson A. Role of diode lasers (800-980 $\mathrm{nm}$ ) as adjuncts to scaling and root planing in the treatment of chronic periodontitis: a systematic review. Photomedicine and laser surgery. 2015;33(11):568575.

6 Moskvin SV. Low-level laser therapy in Russia: history, science and practice. Journal of lasers in medical sciences. 2017;8(2):56.

7 Aoki A, Mizutani K, Schwarz F, Sculean A, Yukna RA, Takasaki AA, et al. Periodontal and peri-implant wound healing following laser therapy. Periodontology 2000. 2015;68(1):217-269.

8 Chaves M. E. D. A., Araújo A. R. D., Piancastelli A. C. C., \& Pinotti M. Effects of low-power light therapy on wound healing: LASER x LED. Anais brasileiros de dermatologia. 2014;89(4):616-623.

9 Cobb CM, Low SB, Coluzzi DJ. Lasers and the treatment of chronic periodontitis. Dental Clinics. 2010;54(1):35-53.

10 Pamuk F, Lütfioğlu M, Aydoğdu A, Koyuncuoglu C, Cifcibasi E, Badur O.The effect of low-level laser therapy as an adjunct to non-surgical periodontal treatment on gingival crevicular fluid levels of transforming growth factor-beta 1, tissue plasminogen activator and plasminogen activator inhibitor 1 in smoking and non-smoking chronic periodontitis patients: A split-mouth, randomized control study.Journal of periodontal research. 2017;52(5):872-882.

11 Gündoğar H, Şenyurt S.Z., Erciyas K, Yalım M,Üstün $\mathrm{K}$. The effect of low-level laser therapy on non-surgical periodontal treatment: a randomized controlled, singleblind, split-mouth clinical trial. Lasers in medical science. 2016;31(9):1767-1773.

12 Roncati M, Gariffo A, Barbieri C, Vescovi P. Ten-Year Nonsurgical Periodontal Treatment Protocol with Adjunctive Use of Diode Laser Monitoring Clinical Outcomes in 6 mm Pockets: A Retrospective Controlled Case Series. International Journal of Periodontics \& Restorative Dentistry. 2017;37(5). nan B, Lakshmi P. A qualitative analysis of periodontal pathogens in chronic periodontitis patients after nonsurgical periodontal therapy with and without diode laser disinfection using Benzoyl-DL arginine-2-naphthylamide test: A randomized clinical trial. Contemporary Clinical Dentistry. 2018;9(3):382.

14 Dukić W, Bago I, Aurer A, Roguljić M. Clinical effectiveness of diode laser therapy as an adjunct to non-surgical periodontal treatment: a randomized clinical study. Journal of periodontology. 2013;84(8):1111-1117.

15 Raj D, Chakrabarty H, Badr J, Das G, Pal T. A comparative evaluation between chlorhexidine chip and diode laser as an adjunct to scaling and root planing in patients with chronic periodontitis. JIDA: Journal of Indian Dental Association. 2017;11(5).

16 Balasubramaniam AS, Thomas LJ, Ramakrishnanan T, Ambalavanan N. Short-term effects of nonsurgical periodontal treatment with and without use of diode laser $(980 \mathrm{~nm})$ on serum levels of reactive oxygen metabolites and clinical periodontal parameters in patients with chronic periodontitis: A randomized controlled trial. Quintessence International. 2014;45(3).

17 Thakare KS, PuriSneha S, BhongadeManohar L. Evaluation of effectiveness of diode LASER for the treatment of periodontal pocket. Indian Journal of Dental Advancements. 2013;5(4):1364-1371.

18 Alzoman H, Diab H. Effect of gallium aluminium arsenide diode laser therapy on $\mathrm{P}$ orphyromonas gingivalis in chronic periodontitis: a randomized controlled trial. International journal of dental hygiene. 2016;14(4):261-266.

19 Saglam M, Kantarci A, Dundar N, Hakki SS. Clinical and biochemical effects of diode laser as an adjunct to nonsurgical treatment of chronic periodontitis: a randomized, controlled clinical trial. Lasers in medical science. 2014;29(1):37-46.
Recibido: 01 de Febrero del 2019

Aceptado: 15 de abril del 2019 